\title{
Grey integrated Multiobjective-Particle Swarm Optimization (MOPSO) for Machining assessment and predictive modeling of Cutting Forces generated during Polymer nanocomposite Drilling
}

\author{
Shivi Kesarwani ${ }^{l}$, Rajesh Kumar Verma ${ }^{2 *}$ \\ ${ }_{1,2}$ Materials and Morphology Laboratory, \\ Department of Mechanical Engineering, Madan Mohan Malaviya University of Technology, Gorakhpur, India-273010 \\ *Corresponding author: rajeshverma.nit@gmail.com
}

\begin{abstract}
Carbon nanomaterials reinforced composite materials have been broadly utilized in manufacturing engineering due to improved thermal resistivity, reduced weight, and other improved mechanical properties. This article highlights the drilling experimentation of zero-dimensional ( 0 -D) Carbon nano onion (CNO) reinforced polymer composite. For this, three drilling constraints was considered viz., spindle speed, feed rate, and weight $\%$ of nanomaterial reinforced. The objective is to achieve the desired value of generated drilling forces such as Torque $(\mathrm{Nm})$ and Thrust Force $(\mathrm{N})$ during the machining procedure of developed composite samples. The Multiobjective-Particle Swarm Optimization (MOPSO) is utilized to achieve optimal results from the multi-decision criterion for the Machining performance. Exploiting this optimization process, non-dominated solutions were obtained, and the Pareto front was identified. Practical applications for the discovered relationships include using Grey relation analysis (GRA) to extract the most relevant finding from the Pareto Front space of optimal solutions. Using the GRA, the optimum solution was found: Spindle Speed of 1000 RPM, Feed Rate of $100 \mathrm{~mm} / \mathrm{min}$, and CNO weight percentage of 0.5. After this, a confirmation test was performed, the expected effects have been confirmed. The findings reveal that the proposed optimization module can be recommended for online quality and productivity control.
\end{abstract}

\section{Introduction}

Polymer materials are cost-effective, lightweight, and have excellent physical-chemical properties. Hence the polymer-based composites are commonly used in customary lifestyle products to critical engineering applications. Polymer nanocomposites become the ideal solution for a new generation complex application [13]. As a result, numerous research work is done on various manufacturing functions related to lightweight and strength uses of nanocomposite materials. In typical polymers, nano-sized fillers have previously been shown to significantly improve both reinforcement properties, such as high aspect ratio and surface to volume ratio. Polymer materials are low-cost, lightweight, and are physically and chemically very strong. Thus, polymer-based composites are widely employed in everyday things because of their critical uses in engineering. Nanocomposites reinforced into the polymers are an excellent solution for a new generation of more complex applications. Moniruzzaman et al. [4] developed an epoxy matrix-based nanocomposite by high shear mixing method with the infusion of 0.01 and 0.05 wt. \% of single-wall carbon nanotube (SWCNT)
Epoxy 862 resin, using dimethylformamide (DMF) as a hardener, concluding that 17 and $10 \%$ improvement in flexural modulus and strength, respectively, over neat resin. Hosur et al. [5] did an intensive study on different development techniques of epoxy /MWCNT nanocomposites, and they revealed that with the addition of 0.2 wt. \% MWCNT with the combining process of sonication, three-roll and inferred that threeroll gives the better mechanical yielded 35.80 and $50.75 \%$ increment in storage modulus at $35^{\circ} \mathrm{C}$, respectively, over the neat epoxy material. Salom et al. [6] have developed graphene-based epoxy nanocomposite material to analyze the mechanical and adhesive behavior of epoxy/Graphene. They conclude that Young's modulus of composite material increased with the GNP concentrations up to $12 \mathrm{Wt}$. \%

Drilling is used in machining operations, and the most frequent of which is cutting away unwanted material to produce products by utilizing rotary parts. MCDM approaches and Metaheuristic optimization techniques are used to identify optimal process parameters, which maximize the positive effect while minimizing the required effort. These parameters govern the drilling process. Several initiatives have been made to improve 
the machining qualities of a drilling process, for example, surface roughness and cutting force. Kharwar and Verma [7] develop the epoxy/MWCNT nanocomposite in different weight concentrations to perform machining operations. The machining characteristics were optimized by hybrid optimization technique Grey Rational Analysis and Principal component analysis. They conclude that the optimized parameter at $1 \mathrm{wt}$. \% for surface integrity, torque and thrust force. In addition, they also revealed that the hybrid optimization technique was more efficient (13.99\%) the single GRA method. Fu et al. [8] performed the micromachining operation on the epoxy/ GNP nanocomposite material and studied the machinability aspects. They analyze the cutting force, surface structure, chip behavior, and deterioration of the tool. They conclude composite with $0.1 \%$ wt. $\%$ attributes the higher mechanical strength and young modulus. Moreover, during the tool study diamond tool gives the higher MRR and best SR comparing to the uncoated tool. Samuel et al. [9] performed the microdrilling of polymer/CNT-based nanocomposites and evaluated the cutting forces, tool deterioration, surface quality, and chip morphology. They also revealed that the average thickness of chips is more significant in the CNT composite with respect to pain polycarbonate composite. Abhishek et al.[10] explained a combined study on Taguchi and PCA fuzzy concept to evaluate a parametric effect of machining response. This approach was proficiently applied for delamination and drilling damages control. The confirmatory findings validated the significance of optimization and modeling of input constrained during the drilling operation of polymeric materials. Gaitonde et al. [11] experimented on an ANN-based model to examine the machining behavior such as specific cutting force, machining force, and power on glass fiber polyamide-based composite. The outcomes depict that feed rate and controlling speed have greatly affected cutting force during the machining. Xu et al. [12] examined the consequence of heat and cutting temperature on high-strength CFR Polymer during the drilling operation. They reveal that the interfacial friction coefficient was highly sensitive among the input variable during the machining operation. Moreover, they established the analytical model for the prediction of output parameters (drilling temperature) under the state of low feed rate. Anand and Patra [13] have performed a micro-drilling operation on CFRP laminates and apply a modeling approach for cutting forces on a mechanistic-based approach during drilling. Researchers validate the modal with experimental thrust force and torque results and find that the average thrust force and torque deviations on the CFRP piles region are $3.99 \%$ and $6.29 \%$, respectively. The same deviations in the matrix region of the composite were $6.30 \%$ and $14.81 \%$, respectively. Mandal et al.[14] utilized hybridization of Technique for order preference by similarity to ideal solution (TOPSIS) Multi-Objective Particle Swarm Optimization (MOPSO) to obtain the best parametric configuration during powder mixed EDM machining of $\mathrm{Cu}-\mathrm{MWCNT}$ composite employing coated 6061Al electrode. The predicted MOPSO-TOPSIS results were compared with experimental data, and it is found to be in good agreement.

Conversely, whereas drilling experiments have studied the machining properties of nanocomposites, these aspects have not been explored in the literature. Few studies have attempted to examine the impact of process parameters on numerous performance measurements, particularly the efficacy of $\mathrm{CNO}$ in bonding to polymer composites reinforced with CNO. The cutting performance of $\mathrm{CNO} /$ Epoxy composites has been studied in the present work. Torque (Tr) and Thrust Force (Th) are two essential reactions evaluated in the current drilling experimentation. It is critical to both theoretical and practical considerations to keep $\mathrm{Tr}$ and Th at a minimum. Optimization and design approaches have proved helpful in improving machining processes. This study presents machining of CNO reinforced epoxy nanocomposite with HSS drilling tool according to BoxBehnken design. Tr and Th were minimized using the MOPSO, the ideal drilling process parameters were determined, and the drilling procedure was optimized with the MOPSO. An optimal set of solutions was found when optimizing with the Pareto Front optimal set. In this article, the GRA process was employed to determine the drilling machining operational status accurately. If the proposed research is successful, the module will provide an enhanced system for monitoring quality control when modifying composite materials. Using swarm movement inspired MOPSO, an attempt has been made to examine the machining efficiency of the $\mathrm{CNO} /$ Epoxy composites.

\section{Experimental Details}

This article projects that the fabrication process of $\mathrm{CNO}$ reinforced epoxy nanocomposites. To fabricate the $\mathrm{CNO} /$ Epoxy composites, the components were compiled and manufactured in two phases. The proper amount of CNO powder has been utilized and dispersed in acetone for 60 minutes employing ultrasonication to avert any agglomeration. Then, in the second phase, the mixture was blended with Epoxy (Lapox L-12) to produce concentrations of $0.5,1.0$, and $1.5 \mathrm{wt} . \%$, after which the mixture was sonicated for 45 minutes. Acetone was separated from the blend through the partial distillation process. This combination of Hardener (Lapox K-6) is then distributed evenly throughout the mixture. The calculated weight of the hardener to the resin was based on the ratio of 1:10 by weight. The mixture is poured into a stainless steel mold with a dimension $100 \mathrm{~mm} \times 100 \mathrm{~mm} \times 10 \mathrm{~mm}$. The molds containing the mixture were permitted to continue curing at room temperature for a period of 24 hours. Henceforth, Casted specimens were taken out from the steel molds. Fig. 1 depicts the process as mentioned earlier.

The drilling experimentation was performed on a Vertical three-axis CNC machine (Make: MAC POWER CNC Machine/Model: V544) setup, as shown in Fig. \#. The experimentation setup has a high-speed spindle of $20,000 \mathrm{rpm}$. The drill bit (HSS, Ø5 mm diameter, Twist drills with point angle $120^{\circ}$ ) assembly 
of the machine tool consists of a chuck. A CNC machine was equipped with a force dynamometer and a data acquisition system for force computations.

The drilling parameters were chosen with restricting compatible cutting conditions in mind. Test runs are used to ensure that the levels and factors are conceivable for the experimental operation. The data collection process has a substantial impact on the results of any experimental inquiry. A full factorial set of tests, in which studies are carried out for all combinations of variables, is the most favored type of experimentation used by researchers. A full factorial DOE (design of experiments) assesses the impact of every possible combination of factors and factor levels.

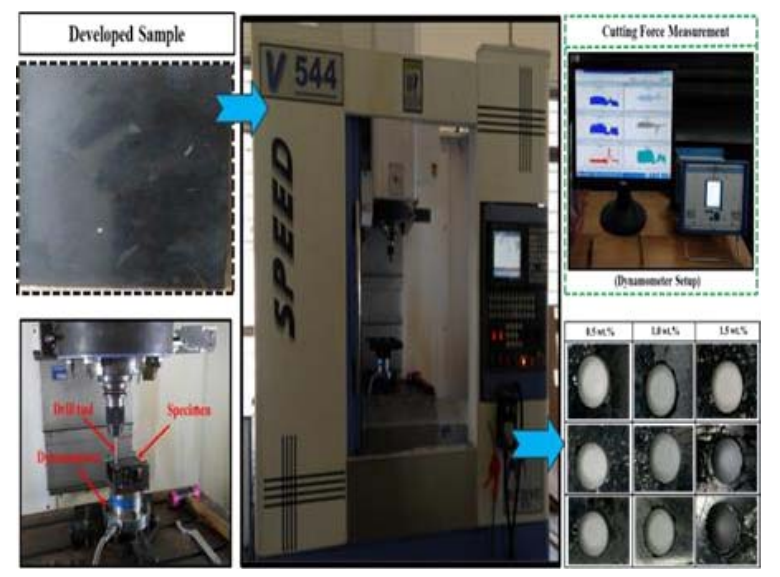

Fig. 1. Experimental setup

The three experimental constraints, precisely $\mathrm{CNO}$ wt.\%, spindle speed, and feed rate, varied at three different Response Surface Methodology (RSM) based Box-Behnken Design (BBD) levels as specified in Table 1. In order to carry out the drilling experiment, the BoxBehnken Design that had 15 runs was utilized. To ensure an accurate and minimal experiment error deviation during experimentation, three iterations of the central experiment were repeated, and the effects of each iteration were observed for 15 experiments in a row.

Table 1 Drilling parameters

\begin{tabular}{|l|l|l|l|}
\hline Drilling Constraints & \multicolumn{3}{|c|}{ Levels } \\
\hline & $\mathbf{1}$ & $\mathbf{2}$ & $\mathbf{3}$ \\
\hline $\boldsymbol{S}_{\boldsymbol{p}}:$ Spindle speed (rpm) & 500 & 1000 & 1500 \\
\hline $\boldsymbol{R}_{\boldsymbol{f}}:$ Feed rate (mm/min) & 50 & 100 & 150 \\
\hline $\boldsymbol{W}_{\boldsymbol{c}}:$ CNO Weight \% & 0.5 & 1.0 & 1.5 \\
\hline
\end{tabular}

Table 2 RSM design and corresponding experimental data

\begin{tabular}{|c|c|c|c|c|c|}
\hline \multirow{2}{*}{$\begin{array}{c}\text { Ex. } \\
\text { No. }\end{array}$} & \multicolumn{2}{|c|}{ Drilling Constraints } & \multicolumn{2}{c|}{ Responses } \\
\cline { 2 - 6 } & $\boldsymbol{S}_{\boldsymbol{p}}$ & $\boldsymbol{R}_{\boldsymbol{f}}$ & $\boldsymbol{W}_{\boldsymbol{c}}$ & $\begin{array}{c}\text { Tr } \\
(\mathbf{N m})\end{array}$ & $\begin{array}{c}\text { Th } \\
(\mathbf{N})\end{array}$ \\
\hline $\mathbf{1 .}$ & 1000 & 100 & 1.0 & 0.329 & 25.844 \\
\hline $\mathbf{2 .}$ & 500 & 100 & 1.5 & 0.389 & 45.460 \\
\hline $\mathbf{3 .}$ & 1000 & 50 & 0.5 & 0.220 & 14.933 \\
\hline $\mathbf{4 .}$ & 1500 & 100 & 0.5 & 0.332 & 21.114 \\
\hline $\mathbf{5 .}$ & 1000 & 150 & 0.5 & 0.343 & 15.256 \\
\hline $\mathbf{6 .}$ & 1500 & 100 & 1.5 & 0.344 & 20.290 \\
\hline $\mathbf{7 .}$ & 500 & 150 & 1.0 & 0.642 & 66.542 \\
\hline
\end{tabular}

\begin{tabular}{|c|c|c|c|c|c|}
\hline 8. & 1500 & 150 & 1.0 & 0.436 & 33.872 \\
\hline 9. & 1500 & 50 & 1.0 & 0.308 & 32.993 \\
\hline $\mathbf{1 0 .}$ & 1000 & 150 & 1.5 & 0.350 & 36.653 \\
\hline $\mathbf{1 1 .}$ & 500 & 50 & 1.0 & 0.525 & 65.663 \\
\hline $\mathbf{1 2 .}$ & 1000 & 100 & 1.0 & 0.311 & 26.844 \\
\hline $\mathbf{1 3 .}$ & 500 & 100 & 0.5 & 0.498 & 36.380 \\
\hline $\mathbf{1 4 .}$ & 1000 & 100 & 1.0 & 0.301 & 22.844 \\
\hline $\mathbf{1 5 .}$ & 1000 & 50 & 1.5 & 0.212 & 39.239 \\
\hline
\end{tabular}

\section{Methodology}

\subsection{Multiobjective-Particle Swarm Optimization (MOPSO)}

In Particle Swarm Optimization (PSO), an array of particles is formed and spread throughout the swarm randomly, which are then used to search a specific set of dimensions for previously located particles. Because of the swarm particles' sociopsychological property of benefiting from the accomplishment of others, the particles have shifted the position vector. These are the personal best coordinates in the problem space $\left(\mathrm{P}_{\text {best }}\right)$. A set of values that coordinates all the particles in the system is used, and the result is called the global best value $\left(\mathrm{G}_{\text {best }}\right)$. At each iteration, the particle position is modified until it obtains the $\mathrm{P}_{\text {best }}$ (best for the particle) and the $\mathrm{G}_{\text {best }}$ (best for the swarm). A particle has velocity and location as two of its components.

$$
\begin{gathered}
k_{i}^{m+1}=w_{p} \cdot k_{1}^{m}+\left(p_{i}-l_{i}^{m}\right) \cdot r_{1} \cdot c_{1} \\
+\left(p_{g}-l_{i}^{m}\right) \cdot r_{2} \cdot c_{2}
\end{gathered}
$$

where, $k_{1}^{m}$ and $l_{i}^{m}$ are the $i^{\text {th }}$ component of particle velocity in swarm space. $k_{i}^{m+1}$ and $l_{i}^{m+1}$ are signifying new components of velocity and ith particle location, respectively. $c_{1}$ and $c_{2}$ are two constructive sociocognitive factors, $\mathrm{w}$ depicts distributive weight, and $r_{l}$ and $r_{2}$ represent random numbers in the range of 0 to 1 . In contrast, $p_{i}$ and $p_{g}$ denotes the individual best and swarm best, respectively. The Multi-Objective Particle Swarm Optimization is an expansion of the original concept, and the MOPSO is utilized to establish domination in solutions. The outputs of MOPSO are held in an external archive of outputs, with solutions that have not been heavily endorsed, called a "repository." Instead of each solution competing for domination over the optimization problem, solutions have emerged on top of one other to form a Pareto front of the problem.

\subsection{Grey Rational Analysis (GRA)}

GRA is one of the most often used strategies for determining the best solution under various conditions. A multi-objective optimization problem is an optimization issue that includes numerous choice qualities and numerous decision alternatives. A GRA seeks to choose the most favorable non-dominant viable alternatives from the set of options. 
Step 1: The experimental data is first to be normalized to avoid variability. Every value is to be given a value between 0 and 1 derived from the original data. Since the response of surface roughness is to be minimized, then smaller the-better characteristics are used for normalization using the following Eq. (3).

$x_{i}^{*}=\frac{x_{i}(k)-\min x_{i}(k)}{\max x_{i}(k)-\min x_{i}(k)}$

And the response of the material removal rate is to be maximized, then larger the-better characteristics are used for normalization using the following Eq. (4).

$x_{i}^{*}=\frac{\max x_{i}(k)-x_{i}(k)}{\max x_{i}(k)-\min x_{i}(k)}$

Where, $i=1 \ldots, \mathrm{m} ; k=1 \ldots, \mathrm{n}, \mathrm{m}$ is the range of experimental information and $\mathrm{n}$ is the range of responses. $X_{i}(k)$ denotes the first sequence, denotes the sequence when the information pre-processing, while $X_{i}(k)$ denotes the biggest worth of $X_{i}(k), \min X_{i}(k)$ denotes the tiniest worth of $X_{i}(k)$, and $\mathrm{x}$ is that the desired worth.

Step 2: Calculation of grey relational coefficient, using Eq. 5.

$\gamma_{i}(k)=\frac{\Delta_{\min }+\gamma \Delta_{\max }}{\Delta_{o i}(k)+\gamma \Delta_{\max }}$

where $\Delta_{o i}$, is the deviation sequence and

$\Delta_{o i}=\left|x_{o}(k)-x_{i}(k)\right|$

where $x_{o}(\mathrm{k})$ denotes the reference sequence and $x_{i}(\mathrm{k})$ termed as comparability sequence.

$\Delta_{\min }$ and $\Delta_{\max }$ are the minimum and maximum values of the absolute differences of all comparing sequences.

Step 3: Finding out the grey relational grade (GRG) as follows:

$G_{i}=\frac{1}{n} \sum_{k=1}^{n} \gamma_{i}(k)$

where, $G_{i}$ is the required grade value and $n=$ number of responses

\section{Result and Discussion}

\subsection{ANOVA for Torque $(\mathrm{Nm})$ and Thrust Force (N)}

Each experimental trial in the current study examined Torque (Tr) and Thrust Force (Th). In Table 2, the measured value of outputs in response to different parametric drilling constraints are illustrated. In this research paper, a mathematical model has been developed using RSM-based BBD methodology for accounting the three response variables to use the model to aid in parametric optimization. ANOVA was applied to assess the influence of the Torque $(\mathrm{Nm})$ and Thrust Force $(\mathrm{N})$. The $P$-value of less than 0.05 is considered during the calculation of the drill parameters interaction, which indicates that these are statistically significant parameters in evaluating drilling parameters. The contribution and mean variation of $\mathrm{Tr}$ and $\mathrm{Th}$ with respect to process constraints are illustrated in Fig. 2.

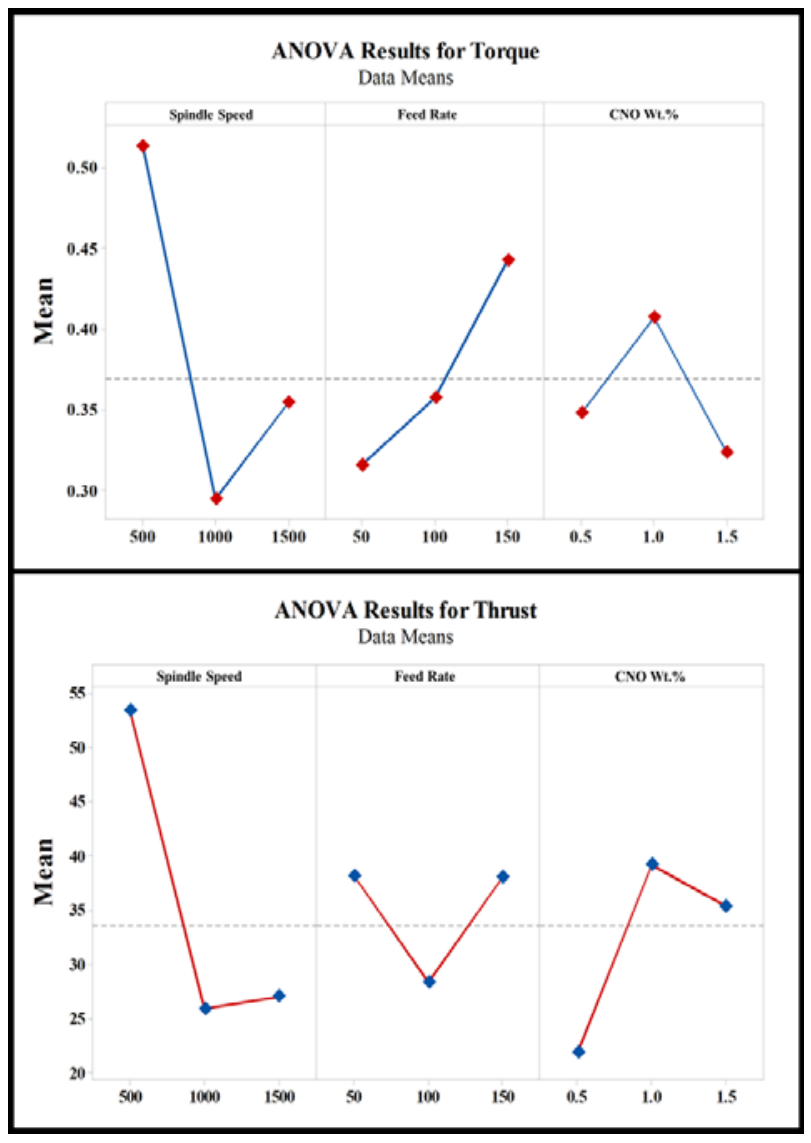

Fig. 2. Analysis of variation for Torque and Thrust Force

The experimental results and forecasted data are analyzed using residual analysis. Fig. 3 shows limitations of drilling constraints related to Torque (Nm) and Thrust Force $(\mathrm{N})$, as well as a standard probability plot of residuals versus percentage. This shows that the experimental points are closer to the standard residuals straight line for both outputs. In Fig.\#, all the values are distributed towards the centerline, indicating that the model is adequate for the confidence interval chosen.

\subsection{Impact of drilling constraints on machining responses}

Fig. 4 and 5 contour plot demonstrates the relationship between drilling torque, polymer composite composition, spindle speed, and feed rate. Similarly, as it could be seen with thrust force, an equivalent pattern appears when discussing drilling torque. This torque pertains to increases in feed rate parallel to a rise in thrust. A change in each of the three $\mathrm{CNO}$ weight \% readings indicates a significant decrease in torque values at a higher spindle speed and a significant rise in torque values at a higher feed rate. The impact of each term is also shown in Fig.; Spindle Speed was found to be the peak contributing factor for Torque $(\mathrm{Nm})$ and Thrust Force $(\mathrm{N})$, whereas the second most contributing factors 
are CNO Wt.\% and Feed Rate found for Thrust Force and Torque, respectively.

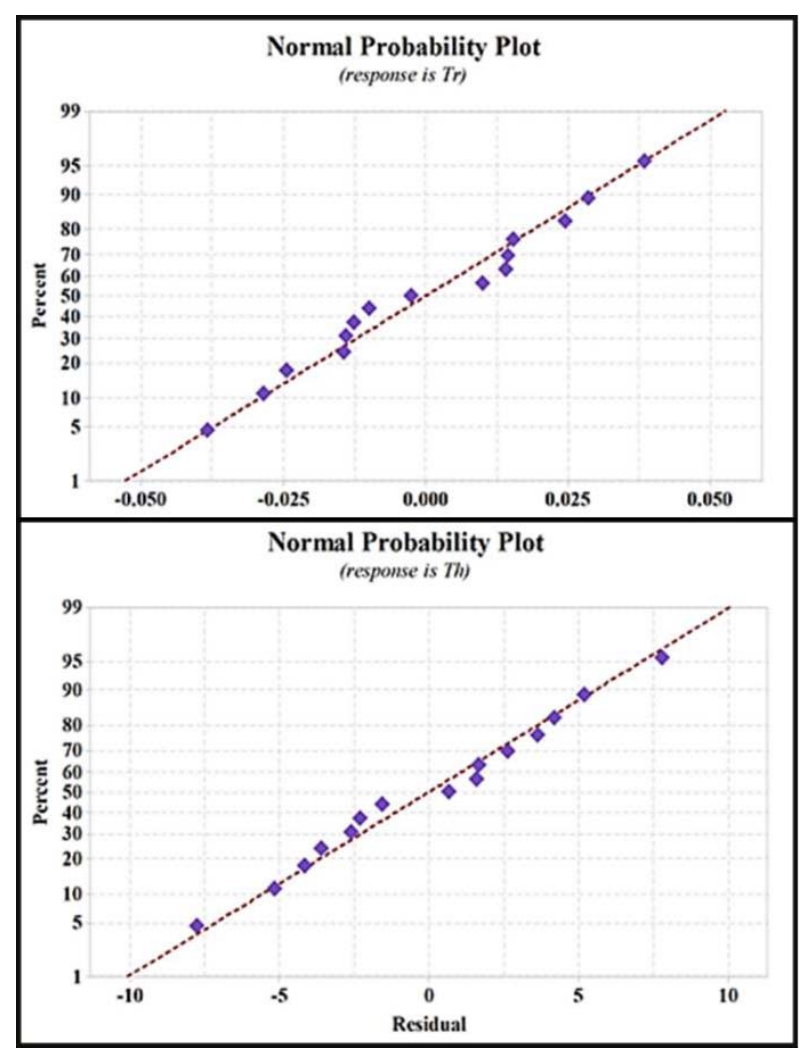

Fig. 3. Residual plot for Torque $(\mathrm{Nm})$ and Thrust Force $(\mathrm{N})$

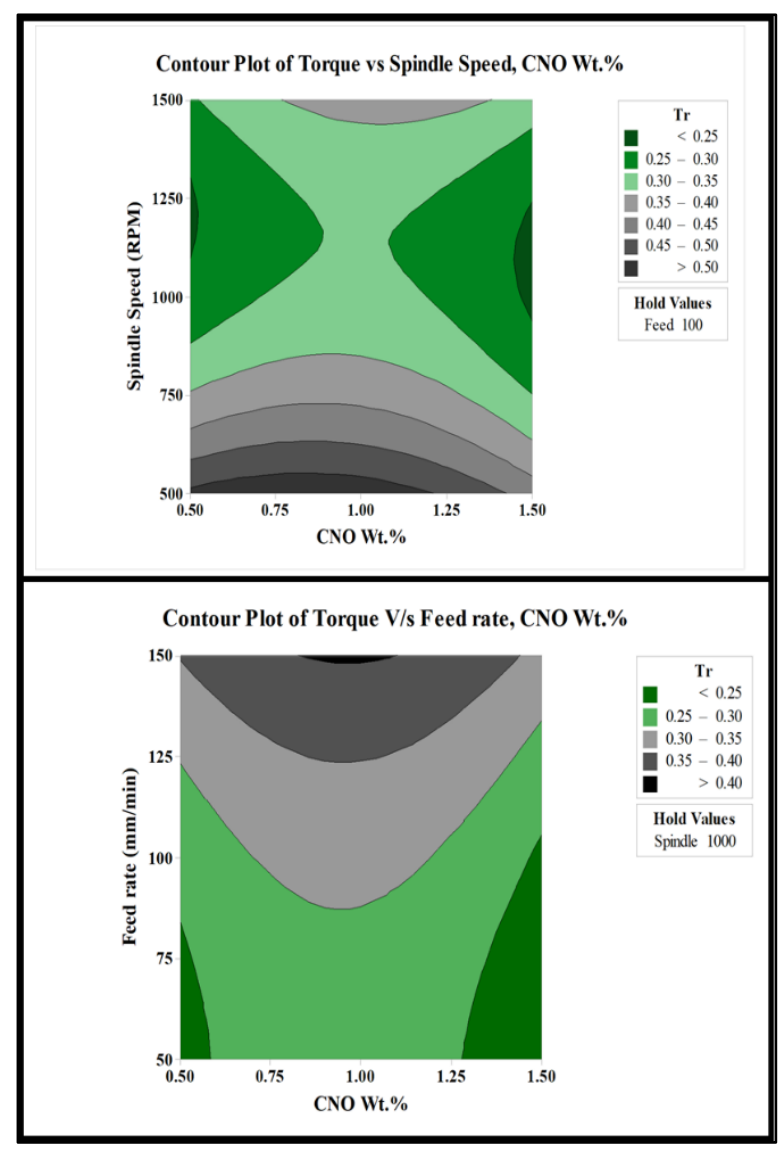

Fig. 4. The contour plot for Torque (Nm)

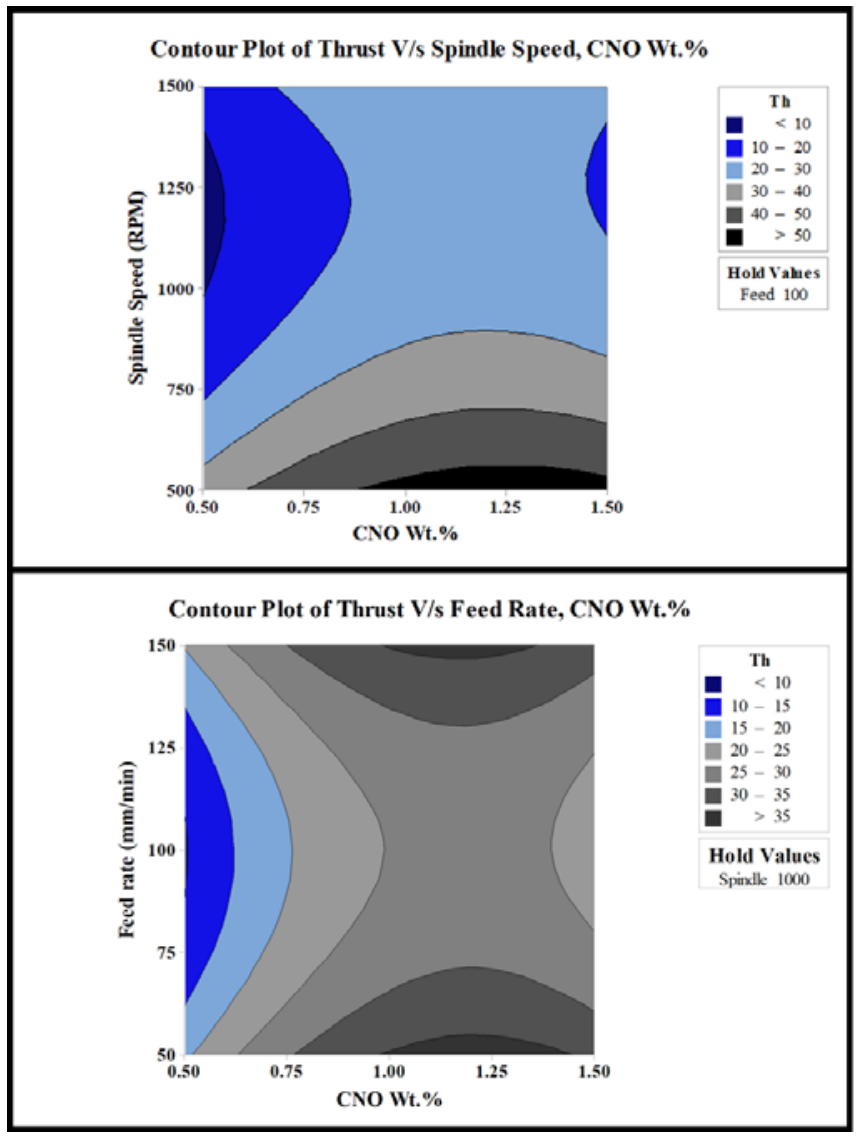

Fig. 5. The contour plot for Thrust Force (N)

\subsection{Mathematical Modelling of Responses}

The response function has been derived using RSM full quadratic polynomial equations from the observed data for Torque $(\mathrm{Nm})$ and Thrust Force $(\mathrm{N})$ by employing statistical analysis software of the Torque and Thrust Force are illustrated as follows.

$$
\begin{aligned}
& \text { Thrust } \quad 88.6-0.1321 \times S_{p}-0.784 \times R_{f} \\
& \text { Force }+96.8 \times W_{c} \\
& (N)=\quad+0.000056 \times S_{p}^{2} \\
& +0.00406 \times R_{f}^{2} \\
& -35.2 \times W_{c}^{2} \\
& -0.0099 \times S_{p} \times W_{c} \\
& -0.029 \times R_{f} \times W_{c} \\
& \boldsymbol{S}=7.259 \quad \boldsymbol{R}^{2}=92.56 \% \quad \boldsymbol{R}^{2}(\text { adj })=79.17 \% \\
& \text { Torque } \\
& (\mathrm{Nm})= \\
& \begin{aligned}
0.935- & 0.001358 \times S_{p} \\
& -0.00118 \times R_{f} \\
& +0.317 \times W_{c} \\
& +0.000001 \times S_{p}^{2} \\
& +0.000011 \times R_{f}^{2} \\
& -0.2388 \times W_{c}^{2} \\
& +0.000121 \times S_{p} \\
& \times W_{c} \\
& +0.000150 \times R_{f} \\
& \times W_{c}
\end{aligned}
\end{aligned}
$$




$$
S=7.25 \quad R^{2}=92.56 \% \quad R^{2}(\text { adj })=79.17 \%
$$

\subsection{Optimization of Torque ( $\mathrm{Nm})$ and Thrust Force (N)}

\subsubsection{Multiobjective-Particle Swarm Optimization (MOPSO)}

In this research article, the global and local search coefficients are taken equally in value and search weight is assigned a value of 0.5 . The MOPSO algorithm values are set to 50,30 , and 150 accordingly to regulate the values of repository size, population size, and iterations. Non-dominated solutions were found in the search space and displayed as red crosses on Pareto space in Fig. 6. It is possible to pick any of the points on the curve, as every point on the curve is the best. At the optimal point, all functions are fulfilled, and it lies somewhere on the Pareto front space.

\subsubsection{Optimal value selection for MOPSO utilizing Grey Relation Analysis (GRA)}

In the MOPSO-GRA technique, solutions obtained from nondomination Pareto front solutions were used. Each objective was given equal weight. The black circle shown on the Pareto front-graph in Fig. \# represents the point at which the GRA approach has found the optimal selection. The information provided in Table 3 demonstrates the values of the goal functions and optimal decision parameters after the GRA process was used.

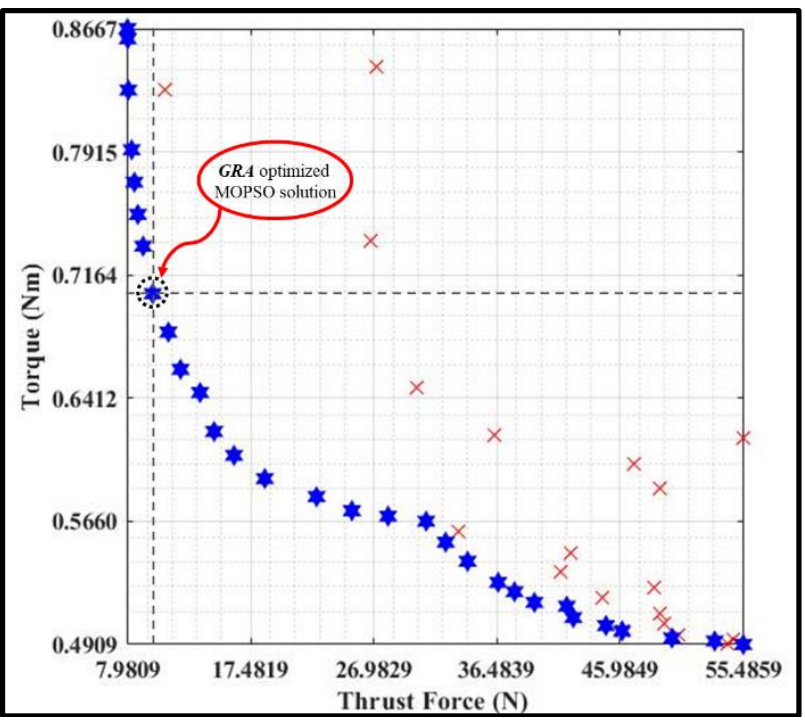

Fig. 6. Pareto Front for Optimal solution obtained using MOPSO with GRA selected optimal point

Table 3. Optimized MOPSO Pareto front with GRA criterion set

\begin{tabular}{|c|c|c|c|c|}
\hline \multicolumn{3}{|c|}{ Drilling Constraints } & \multicolumn{2}{c|}{ Output Responses } \\
\hline $\begin{array}{c}\boldsymbol{S}_{\boldsymbol{p}} \\
(\mathbf{R P M})\end{array}$ & $\begin{array}{c}\boldsymbol{R}_{\boldsymbol{f}} \\
(\mathbf{m m} / \mathbf{m i n})\end{array}$ & $\begin{array}{c}\boldsymbol{W}_{\boldsymbol{c}} \\
(\mathbf{C N O} \\
\mathbf{W t} \mathbf{\%})\end{array}$ & $\begin{array}{c}\text { Torque } \\
(\mathbf{N m})\end{array}$ & $\begin{array}{c}\text { Thrust } \\
(\mathbf{N})\end{array}$ \\
\hline 1000 & 100 & 0.50 & 0.705 & 9.926 \\
\hline
\end{tabular}

\subsection{Confirmatory Test}

For the confirmatory experimentation, two objectivebased modules were estimated to obtain the optimal input parameter settings by employing MOPSO-GRA. The predicted settings were compared to confirm the effectiveness of the output responses obtained experimentally. The results of the confirmation test and error percentages are listed in Table 4 . The Torque $(\mathrm{Nm})$ and Thrust Force $(\mathrm{N})$ error percentages are each found to be 8.630 and 3.360 , respectively. Based on the experiments, it can be concluded that the MOPSO-GRA methodology was proven valid, and the technique can be utilized to provide estimates for milling process parameters.

Table 4: Confirmatory test results

\begin{tabular}{|c|c|c|c|c|}
\hline $\begin{array}{c}\text { Sr. } \\
\text { No. }\end{array}$ & $\begin{array}{c}\text { Output } \\
\text { Responses }\end{array}$ & $\begin{array}{c}\text { Predicted } \\
\text { Results }\end{array}$ & $\begin{array}{c}\text { Experimental } \\
\text { Results }\end{array}$ & $\begin{array}{c}\text { Error } \\
\%\end{array}$ \\
\hline $\mathbf{1}$ & $\begin{array}{c}\text { Torque } \\
\text { (Nm) }\end{array}$ & 0.705 & 0.644 & 8.630 \\
\hline $\mathbf{2}$ & $\begin{array}{c}\text { Thrust } \\
\text { (N) }\end{array}$ & 9.926 & 9.592 & 3.360 \\
\hline
\end{tabular}

\section{Conclusion}

In the present study, the $\mathrm{CNO} /$ Epoxy composites were machined employing drilling by varying process constraints. The RSM-based Box-Behnken Design was utilized to formulate the design of experiment (DOE) array. A full quadratic $2^{\text {nd }}$ order polynomial regression equations model was formed by analytically analyzing experimentation responses. Additionally, the MOPSO approach was used to optimize the drilling process parameters based on fitness functions. In this optimization problem, two functions were the objective: Torque $(\mathrm{Nm})$ and Thrust Force $(\mathrm{N})$. It is critical to minimize both. In the Pareto optimum front design space, the range of non-dominated optimal solutions for Torque (Nm) and Thrust Force $(\mathrm{N})$ has 7.981 to 55.486 and 0.491 to 0.8667 , respectively. Using the GRA approach, the best possible solution was chosen, and each objective function was weighted equally during the application of this technique. Torque and Thrust Force measured during drilling testing yielded the optimal results and were observed to be $0.705 \mathrm{Nm}$, and $9.926 \mathrm{~N}$. Optimal drilling constraints $\left(\boldsymbol{S}_{\boldsymbol{p}}=1000 \mathrm{rpm}, \boldsymbol{R}_{\boldsymbol{f}}=100\right.$ $\mathrm{mm} / \mathrm{min}$, and $\boldsymbol{W}_{\boldsymbol{c}}=0.5 \%$ ) were found for this process result. After getting the solutions from the MOPSOGRA, these results were validated with a confirmation test, and a positive result was obtained. The finding of the proposed hybrid module can be used for other machining operations such as milling, turning, etc. Also, it can be customized for different case studies of multiple criteria optimization of industrial engineering.

\section{Acknowledgment}

The authors would like to acknowledge the Uttar Pradesh Council of Science and Technology, Lucknow under Govt. of Uttar Pradesh, India. 


\section{Funding}

This presented work is financially supported by the Uttar Pradesh Council of Science and Technology, Lucknow, India, under the research project scheme [R\&D project ID-UPCST/ D-2491].

\section{References}

1. Kyzas, G. Z. Compos. Interfaces 25, 415 (2018).

2. Keyte, J., Pancholi, K. \& Njuguna, J. Recent Front. Mater. 6, 1(2019).

3. Pikhurov, D. V. \& Zuev, V. V., Chem. Phys. Lett. 601, 13(2014).

4. Moniruzzaman, M., Du, F., Romero, N. \& Winey, K. I. Polymer (Guildf). 47, 293 (2006).

5. Hosur, M. et al. J. Appl. Polym. Sci. 127, 4211 (2013).

6. Salom, C. et al. Int. J. Adhes. Adhes. 84, 119 (2018).

7. Kumar Kharwar, P. \& Kumar Verma, R.. Measurement 158, 107701 (2020).

8. Fu, G., Huo, D., Shyha, I., Pancholi, K. \& Alzahrani, B. Int. J. Adv. Manuf. Technol. 107, 3169 (2020).

9. Samuel, J., DeVor, R. E., Kapoor, S. G. \& Hsia, K. J. J. Manuf. Sci. Eng. Trans. ASME 128, 465 (2006).

10. Abhishek, K., Datta, S. \& Mahapatra, S. S. Int. J. Adv. Manuf. Technol. 76, 401(2014).

11. Gaitonde, V. N., Karnik, S. R., Mata, F. \& Davim, J. P., J. Thermoplast. Compos. Mater. 23, 313-336 (2010).

12. $\mathrm{Xu}$, J., Li, C., Dang, J., El Mansori, M. \& Ren, F. Materials (Basel). 11, (2018).

13. Anand, R. S. \& Patra, K. CIRP J. Manuf. Sci. Technol. 16, 55-63 (2017).

14. Mandal, P. \& Mondal, S. C. Meas. J. Int. Meas. Confed. 169, 108347 (2021). 